\title{
The Zwicky Transient Facility
}

Eric Bellm

California Institute of Technology

\section{Introduction}

The Zwicky Transient Facility (ZTF; P.I. Shri Kulkarni) is a next-generation optical synoptic survey that builds on the experience and infrastructure of the Palomar Transient Factory (PTF) $[12,18]$. Using a new $47 \mathrm{deg}^{2}$ survey camera, ZTF will survey more than an order of magnitude faster than PTF to discover rare transients and variables.

PTF (and its successor survey, the Intermediate Palomar Transient Factory, or iPTF) have conducted a transient-focused optical time-domain survey. PTF uses a $7.26 \mathrm{deg}^{2}$ camera on the Palomar 48-inch Oschin Schmidt telescope (P48) to survey the dynamic night sky in Mould- $R$ and SDSS $g^{\prime}$ bands. Followup photometry and spectroscopy are provided by the 60- and 200-inch telescopes at Palomar and by other collaboration resources around the world.

PTF's moderate-depth, followup-focused survey has yielded many notable successes. However, addressing leading-edge scientific questions (Section 4) requires a capability to survey at high cadence while maintaining wide areal coverage. Current facilities are inadequate for this purpose, but a straightforward upgrade of the PTF survey camera provides this capability while maintaining much of PTF's demonstrably productive hardware and software infrastructure. ZTF will provide the best characterization of the bright to moderate-depth $(m \lesssim 21)$ transient and variable sky and pave the way for LSST's deeper survey.

\section{Survey Design}

The traditional measure of étendue (collecting area $\times$ solid angle) is insufficient for characterizing the performance of time-domain surveys [22]. It relates most closely to the speed at which an instrument achieves a given coadded depth. Time domain surveys are often interested in the detection rate for specific classes of transients (e.g., Type Ia SNe or Tidal Disruption Events). These detection rates are a function of the intrinsic rate, brightness, and timescale of the transient; the cadence of the survey; and the spatial volume surveyed in each cadence period. For variability science, 
the utility of time series data depends on the limiting magnitude, the photometric precision, the total number of observations, the cadence, and the bandpass(es) of the data.

This wide range of survey parameter space indicates the difficulty of optimizing a generic time-domain survey for a wide range of science goals. (It also suggests that specialized surveys will continue to be productive into the era of large timedomain facilities.) In consequence, single figures of merit are imperfect predictors of the performance of a time-domain survey, as much depends on the specifics of the chosen survey strategy in addition to the raw capabilities of the camera and telescope. However, optimization metrics are required to guide design studies and cost trades.

Building on the PTF heritage, we have chosen to optimize the ZTF camera design for the study of explosive transients. For any camera realization, we may trade survey cadence against the sky area covered per survey snapshot. We therefore seek to maximize the volumetric survey rate $(\dot{V})$, defined as the spatial volume within which a transient of specified absolute magnitude (e.g, $M=-19$ ) could be detected at $5 \sigma$, divided by the total time per exposure including readout and slew times. With appropriate choice of cadence, $\dot{V}$ should be proportional to the transient detection rate. It implicitly incorporates the field of view of the camera, its limiting magnitude (which in turn includes the image quality, sky background, telescope and filter throughput, and read noise), and overheads [c.f. 22].

Notably, specifying the overhead between exposures implies an optimal exposure time to maximize $\dot{V}$. Exposures that are too short lead to an inefficient duty cycle, while exposures that are too long lead to smaller snapshot volumes, as the loss of areal covered is not offset by the increase in depth.

Guided by these considerations, our design for the ZTF survey camera (Section 3) maximizes the camera field of view, maintains PTF's moderate image quality and depth, and minimizes the overhead between exposures and the number of filters.

\section{The ZTF Camera}

The $7.26 \mathrm{deg}^{2}$ field of view provided by the CFHT12k camera [17] currently used by PTF only covers a fraction of the available $\sim 47 \mathrm{deg}^{2}$ focal surface of the P48. By constructing a new camera that fills the focal surface with CCDs, we obtain a 6.5 times larger field of view. Modern readout electronics will reduce the overhead between exposures as well, providing a net improvement in survey speed of more than an order of magnitude relative to PTF. This speed boost will enable a transformative survey capable of simultaneously maintaining the high cadence and wide areal coverage needed to find rare, fast, and young transients.

The focal surface of the Schmidt telescope is curved, and during the Palomar Sky Surveys the photographic plates were physically bent on a mandrel to conform to 


\begin{tabular}{|l|r|r|}
\hline Specification & PTF & ZTF \\
\hline Active Area & $7.26 \mathrm{deg}^{2}$ & $47 \mathrm{deg}^{2}$ \\
Exposure Time & $60 \mathrm{sec}$ & $30 \mathrm{sec}$ \\
Readout Time & $36 \mathrm{sec}$ & $10 \mathrm{sec}$ \\
Median Time Between Exposures & $46 \mathrm{sec}$ & $15 \mathrm{sec}$ \\
Median Image Quality $(R$ band $)$ & $2.0 " \mathrm{FWHM}$ & $2.0 " \mathrm{FWHM}$ \\
Median Single-visit Depth $(R$ band $)$ & 20.7 & 20.4 \\
Yearly Exposures per Field $(3 \pi)$ & 19 & 290 \\
Areal Survey Rate & $247 \mathrm{deg}^{2} / \mathrm{hr}$ & $3760 \mathrm{deg}^{2} / \mathrm{hr}$ \\
Volumetric Survey Rate $(M=-19)$ & $2.8 \times 10^{3} \mathrm{Mpc}^{3} / \mathrm{s}$ & $3.0 \times 10^{4} \mathrm{Mpc}^{3} / \mathrm{s}$ \\
\hline
\end{tabular}

Table 1: Comparison of the PTF and ZTF cameras and survey performance metrics. Yearly exposures assume a hypothetical uniform $3 \pi$ survey.

this focal surface. The PTF camera achieves acceptable image quality (median 2" FWHM in $R$ ) with a flat CCD focal plane, an optically powered dewar window, and flat filters. However, scaling a comparable design to the full ZTF focal plane produces unacceptable image quality.

We have developed an optical design that maintains PTF's image quality over the entire available field of view. An additional zero-power optic (to be fabricated from an existing blank) placed in front of the existing achromatic doublet Schmidt corrector provides a minor adjustment $(10 \%)$ to its aspheric coefficient. A faceted CCD focal plane and individual field flattener lenses placed over each CCD correct the residual field curvature. An optically powered window maintains vacuum in the dewar. The optical design supports exchangeable flat filters, or the filter coatings may be deposited onto the field flatteners mounted over each CCD.

Improved yields for wafer-scale CCDs make large focal planes increasingly affordable. ZTF will use $16 \mathrm{e} 2 \mathrm{v} 6 \mathrm{k} \times 6 \mathrm{k}$ devices with 15 micron pixels. At 1"/pixel, the pixel scale is identical to that of PTF and adequately samples the median 2" image quality. The moderate pixel scale also mitigates the data volume. Six CCDs have been fabricated and delivered as of this writing. At $1 \mathrm{MHz}$ readout, read time will be $10 \mathrm{sec}$; we require $15 \mathrm{sec}$ net overhead between exposures to allow for slew and settling. With these shorter overheads, $30 \mathrm{sec}$ exposures are optimal in maximizing $\dot{V}$. A compact dewar design minimizes mass and beam obstruction.

Table 1 compares the performance of the ZTF survey camera to that of PTF. 


\section{Selected Science Goals}

\subsection{Young SNe}

Observations of SNe within the first 24 hours of explosion reveal key information about their progenitors and environments. Early photometric observations of SNe Ia constrain the radius of the progenitor and can distinguish single- and double-degenerate scenarios [7]. In core-collapse $\mathrm{SNe}$, early observations probe the poorly-measured physics of shock breakout and shock heating [16]. Early-time "flash" spectroscopy of core-collapse SNe within hours of the explosion can directly measure the properties of the circumstellar medium and reveal the final stages of stellar evolution before the explosion [5].

Detecting, discovering, and following up young transients in a single night requires finely honed pipelines, procedures, and collaboration. The PTF and iPTF collaborations have demonstrated the ability to obtain these time-critical measurements on several occasions [6]. However, the total number of young SNe in the PTF datastream is limited by the survey camera: obtaining the few-hour cadence observations needed to detect young SNe limits the survey to a much smaller area of sky. With ZTF's wider, faster camera, the collaboration will be able to systematically study a true sample of SN progenitors rather than an isolated handful: we can detect twelve times more SNe with ZTF at any chosen cadence. In a high-cadence survey, ZTF will detect one SN within 24 hours of its explosion every night.

\subsection{Fast-decaying transients}

While PTF, CRTS, and Pan-STARRS1 have occasionally observed at relatively high cadences (images separated by less than a few hours), the correspondingly small areal coverage permitted by their survey cameras has limited the detection of fast transients to M-dwarf flares [1]. ZTF's order-of-magnitude increase in survey speed will place much tighter constraints on the existence of fast-decaying explosive transients, exceeding published limits on areal exposure in less than one week of observations.

One intriguing event, PTF11agg [3], highlights the potential of ZTF in this area. Discovered by PTF during high-cadence monitoring of the Beehive Cluster for variable star studies, PTF11agg declined by almost two magnitudes over several hours. While its properties are consistent with an optical afterglow of a gamma-ray burst (GRB), there was no high-energy trigger from wide-field gamma-ray monitor. This raises the possibility that PTF11agg represents a new class of event, a baryon-loaded "dirty fireball" that would not show MeV emission. The inferred rate of such events would be about twenty times the GRB rate.

With ZTF's faster survey speed, we expect to detect more than 20 PTF11agglike events per year, as well as a handful of classical GRB orphan optical afterglows. 
These measurements will place important constraints on the opening angles of GRB jets as well as the diversity of relativistic stellar explosions.

\subsection{Gravitational Wave Counterparts}

Beginning in 2015, advanced gravitational wave (GW) interferometers will begin operations. They are expected to detect the first GW signals from binary neutron star mergers. Detecting the electromagnetic counterparts to these events will provide vital physical information, including independent distance estimates and information about the merger progenitors and host galaxy. The mergers are predicted to produce optical counterparts, whether from afterglows of short-hard gamma-ray bursts or "kilonovae" powered by r-process nucleosynthesis [13, 11, 14, 8].

Unfortunately, the earliest GW detections will be very poorly localized, with error boxes of hundreds of square degrees with only two detectors and improving to tens of square degrees as more interferometers come online. Detecting a rapidly-decaying optical transient with unknown brightness in this large sky area is a monumental challenge. Success will require a well-tested technical stack, including all-sky reference images, fast and reliable image differencing, a complete local galaxy catalog to prioritize followup, and the ability to obtain rapid spectroscopy [9]. iPTF has proven this approach by successfully using its transient pipeline to localize the afterglows of Fermi-detected gamma-ray bursts within 70 square degrees [21]. ZTF's wider field will be vital for achieving the same success with the larger search areas and fainter counterparts provided by GW detections.

\subsection{Variability Science}

The repeated observations provided by PTF and other surveys have built an increasingly valuable photometric variability catalog. Single-filter time variability information may be used to identify and classify variable stars [19], identify binary systems, and measure the mass of the supermassive black holes in AGN systems [10]. Variable stars may be used to trace Galactic structure and identify dwarf galaxies [4, 20], thereby mapping the gravitational potential of the Milky Way and testing predictions of $\Lambda$ CDM cosmology [2]. Photometric variability may even predict stellar parameters, including effective temperature, surface gravity, and metallicity [15].

ZTF's greater survey speed will provide an unprecedented variability catalog. If observations are spread evenly over the entire visible Northern sky, we will obtain nearly 300 observations per field each year. CRTS currently provides the most uniform photometric variability coverage. ZTF will provide a larger number of observations as well as improved cadence and depth, enabling a wide range of variability science on sources accessible to moderate-aperture telescopes and advancing community involvement in advance of LSST's deeper survey. 
E. B. is grateful for useful conversations with Shri Kulkarni, Tom Prince, Richard Dekany, Roger Smith, Jason Surace, Eran Ofek, Mansi Kasliwal, Branimir Sesar, and Paul Groot. 


\section{References}

[1] Berger, E., Leibler, C. N., Chornock, R., et al. 2013, ApJ, 779, 18

[2] Bullock, J. S., \& Johnston, K. V. 2005, ApJ, 635, 931

[3] Cenko, S. B., Kulkarni, S. R., Horesh, A., et al. 2013, ApJ, 769, 130

[4] Drake, A. J., Catelan, M., Djorgovski, S. G., et al. 2013, ApJ, 765, 154

[5] Gal-Yam, A. 2014, in American Astronomical Society Meeting Abstracts, Vol. 223, American Astronomical Society Meeting Abstracts, \#235.02

[6] Gal-Yam, A., Kasliwal, M. M., Arcavi, I., et al. 2011, ApJ, 736, 159

[7] Kasen, D. 2010, ApJ, 708, 1025

[8] Kasen, D., Badnell, N. R., \& Barnes, J. 2013, ApJ, 774, 25

[9] Kasliwal, M. M., \& Nissanke, S. 2013, ArXiv e-prints, arXiv:1309.1554 [astroph.HE]

[10] Kelly, B. C., Bechtold, J., \& Siemiginowska, A. 2009, ApJ, 698, 895

[11] Kulkarni, S. R. 2005, ArXiv Astrophysics e-prints, arXiv:astro-ph/0510256

[12] Law, N. M., Kulkarni, S. R., Dekany, R. G., et al. 2009, PASP, 121, 1395

[13] Li, L.-X., \& Paczyński, B. 1998, ApJL, 507, L59

[14] Metzger, B. D., Martínez-Pinedo, G., Darbha, S., et al. 2010, MNRAS, 406, 2650

[15] Miller, A., Richards, J., Bloom, J. S., \& on behalf of a larger team. 2014, in American Astronomical Society Meeting Abstracts, Vol. 223, American Astronomical Society Meeting Abstracts, \#125.01

[16] Nakar, E., \& Sari, R. 2010, ApJ, 725, 904

[17] Rahmer, G., Smith, R., Velur, V., et al. 2008, in Society of Photo-Optical Instrumentation Engineers (SPIE) Conference Series, Vol. 7014, Society of PhotoOptical Instrumentation Engineers (SPIE) Conference Series

[18] Rau, A., Kulkarni, S. R., Law, N. M., et al. 2009, PASP, 121, 1334

[19] Richards, J. W., Starr, D. L., Miller, A. A., et al. 2012, ApJS, 203, 32

[20] Sesar, B., Grillmair, C. J., Cohen, J. G., et al. 2013, ApJ, 776, 26

[21] Singer, L. P., Cenko, S. B., Kasliwal, M. M., et al. 2013, ApJ, 776, L34

[22] Tonry, J. L. 2011, PASP, 123, 58 